\title{
Partonic Picture of Nuclear Shadowing at Small $x^{*}$
}

\section{Ina Sarcevic ${ }^{\mathrm{a}}$}

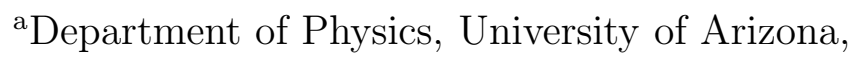
Tucson, AZ 85721

We find that coherent multiple scatterings of the partonic fluctuations of the virtual photon with the nucleons inside the nucleus result in the large nuclear shadowing effect for parton distributions in nuclei. We predict the gluon shadowing effect at small $x$ and for $Q^{2} \geq 3 \mathrm{GeV}^{2}$.

The theoretical studies of semihard processes play an increasingly important role in ultrarelativistic heavy-ion collisions at $\sqrt{s} \geq 200 \mathrm{GeV}$ in describing the global collision features such as particle multiplicities and transverse energy distributions. These semihard processes can be reliably calculated in the framework of perturbative QCD and they are crucial for determining the initial condition for the possible formation of a quark-gluon plasma. Assuming the validity of the factorization theorem in perturbation theory, it is essential to know the parton distributions in nuclei in order to compute these processes.

The attenuation of quark density in a nucleus has been firmly established experimentally at CERN [1] and Fermilab [2] in the region of small $x$ in deeply-inelastic lepton scatterings (DIS) off nuclei. The data, taken over a wide kinematic range, $10^{-5}<x<0.1$ and $0.05 \mathrm{GeV}^{2}<Q^{2}<100 \mathrm{GeV}^{2}$, show a systematic reduction of nuclear structure function $F_{2}^{A}\left(x, Q^{2}\right) / A$ with respect to the free nucleon structure function $F_{2}^{N}\left(x, Q^{2}\right)$. There are also some indications of nuclear gluon shadowing from the analysis of $\mathrm{J} / \Psi$ suppression in hadron-nucleus experiments [3] but the extraction of nuclear gluon density is not unambiguous since it involves the evaluation of the initial parton energy loss and final state interactions [4].

At low $Q^{2}$, in DIS, the interaction of the virtual photon with the nucleons in the rest frame of the target is most naturally described by a vector-meson-dominance (VMD) model [5]. At $Q^{2}>1 \sim 2 \mathrm{GeV}^{2}$, the virtual photon can penetrate the nucleon and probe the partonic degrees of freedom where a partonic interpretation based on perturbative QCD is most relevant in the infinite momentum frame. In the target rest frame, the virtual photon interacts with nucleons via its quark-antiquark pair $(q \bar{q})$ color-singlet fluctuation [6]. If the coherence length of the virtual photon is larger than the distance between nucleons in a nucleus, $l_{c}>R_{N N}$, the $q \bar{q}$ configuration interacts coherently with fraction of the nucleons, while for $l_{c}>R_{A}$ (i.e. $x<10^{-2}$ ), it interacts coherently with all the nucleons, with a cross section given by the color transparency mechanism for a point-like

\footnotetext{
*This work was done in collaboration with Z. Huang and H.J. Lu and was supported in part by the U.S. Department of Energy Contract No. DE-FG03-93ER40792.
} 
color-singlet configuration, $\sigma_{q \bar{q} N}=\frac{4 \pi^{2}}{3} r_{t}^{2} \alpha_{s} x^{\prime} g_{\text {DLA }}\left(x^{\prime}, 1 / r_{t}^{2}\right)$ [8]. This results in a reduction of the total cross section and consequently attenuation of the parton distributions in nuclei.

In the Glauber-Gribov multiple-scattering theory [7] nuclear collision is a succession of collisions of the probe with individual nucleons within nucleus. A partonic system $(h)$, being a $q \bar{q}$ or a $g g$ fluctuation, can scatter coherently from several or all nucleons during its passage through the target nucleus. The interference between the multiple scattering amplitudes causes a reduction of the $h A$ cross section compared to the naive scaling result of $A$ times the respective $h N$ cross section, the origin of the nuclear shadowing. The total $h A$ cross section is given by

$$
\sigma_{h A}=\int d^{2} \mathbf{b} 2\left[1-e^{-\sigma_{h N} T_{A}(\mathbf{b}) / 2}\right]=2 \pi R_{A}^{2}\left[\gamma+\ln \kappa_{h}+E_{1}\left(\kappa_{h}\right)\right],
$$

where $\kappa_{h}=A \sigma_{h N} /\left(2 \pi R_{A}^{2}\right)$ is an impact parameter averaged effective number of scatterings. For small value of $\kappa_{h}, \sigma_{h A} \rightarrow 2 \pi R_{A}^{2} \kappa_{h}=A \sigma_{h N}$, the total $h A$ cross section is proportional to $A$. In the limit $\kappa_{h} \rightarrow \infty$, the destructive interference between multiple scattering amplitudes reduces the cross section, $\sigma_{h A} \rightarrow 2 \pi R_{A}^{2}\left(\gamma+\ln \kappa_{h}\right)$. Namely, the effective number of scatterings is large and the total cross section approaches the geometric limit $2 \pi R_{A}^{2}$, a surface term which varies roughly as $A^{2 / 3}$.

In the Glauber-Gribov eikonal approximation,

$$
\sigma\left(\gamma^{\star} A\right)=\int d^{2} b \int_{0}^{1} d z \int d^{2} \mathbf{r}|\psi(z, \mathbf{r})|^{2} 2\left(1-e^{-\sigma_{q \bar{q} N}(\mathbf{r}, z) T_{A}(b) / 2}\right),
$$

where $|\psi(z, \mathbf{r})|$ is the photon wave function [10] and $z$ is the fraction of the energy carried by the quark (antiquark). The nuclear cross section is therefore reduced when compared to the simple addition of free nucleon cross sections.

At small $x$, the structure function of a nucleus, $F_{2}^{A}\left(x, Q^{2}\right)$ can be obtained from $\sigma\left(\gamma^{*} A\right)$. Substituting integration over $\left(z, \mathbf{r}_{\mathbf{t}}\right)$ to $\left(x^{\prime}, Q^{\prime 2}\right)$ in $(2)$, one obtains for (sea)quarks [9]

$$
x f_{A}\left(x, Q^{2}\right)=x f_{A}\left(x, Q_{0}^{2}\right)+\frac{3 R_{A}^{2}}{8 \pi^{2}} x \int_{x}^{1} \frac{d x^{\prime}}{x^{\prime 2}} \int_{Q_{0}^{2}}^{Q^{2}} d Q^{\prime 2}\left[\gamma+\ln \left(\kappa_{q}\right)+E_{1}\left(\kappa_{q}\right)\right]
$$

where $\kappa_{q}\left(x, Q^{2}\right)=\frac{2 A \pi}{3 R_{A}^{2} Q^{2}} \alpha_{s}\left(Q^{2}\right) x g_{N}^{\mathrm{DLA}}\left(x, Q^{2}\right)$. We find our result for $F_{2}^{N}\left(x, Q^{2}\right)$ to be in excellent agreement with the recent HERA data for a broad range of $x$ and $Q^{2}$ [9].

We parametrize the initial shadowing ratio $R_{0}^{q}(x)$ at $Q_{0}^{2}=0.4 \mathrm{GeV}^{2}$ using experimental data and we calculate the nuclear structure function at the measured $\left\langle Q^{2}\right\rangle$ values at different $x$ values. The results for ${ }^{40} \mathrm{Ca}$ and ${ }^{208} \mathrm{~Pb}$ are shown in Fig. 1 . We note that at large $Q^{2}$ the nuclear shadowing effect is reduced but does not diminish, i.e. it is not a higher twist effect. This can be understood as the interplay between the perturbative and the non-perturbative shadowing mechanisms, which is also evident in the $x$-dependence of $R_{q}$ [9]. The apparent flatness of the shadowing ratio at low $Q^{2}$ in the small- $x$ region is altered by the perturbative evolution. This is due to the singular behavior of $x g_{N}^{\text {DLA }}$ as $x \rightarrow 0$ at large $Q^{2}$ leading to the strong $x$-dependence of the effective number of scatterings, $\kappa_{q}(x)$.

Similarly, gluon distribution in a nucleus is given by

$$
x g_{A}\left(x, Q^{2}\right)=x g_{A}\left(x, Q_{0}^{2}\right)+\frac{2 R_{A}^{2}}{\pi^{2}} \int_{x}^{1} \frac{d x^{\prime}}{x^{\prime}} \int_{Q_{0}^{2}}^{Q^{2}} d Q^{\prime 2}\left[\gamma+\ln \left(\kappa_{g} \tau\right)+E_{1}\left(\kappa_{g} \tau\right)\right]
$$




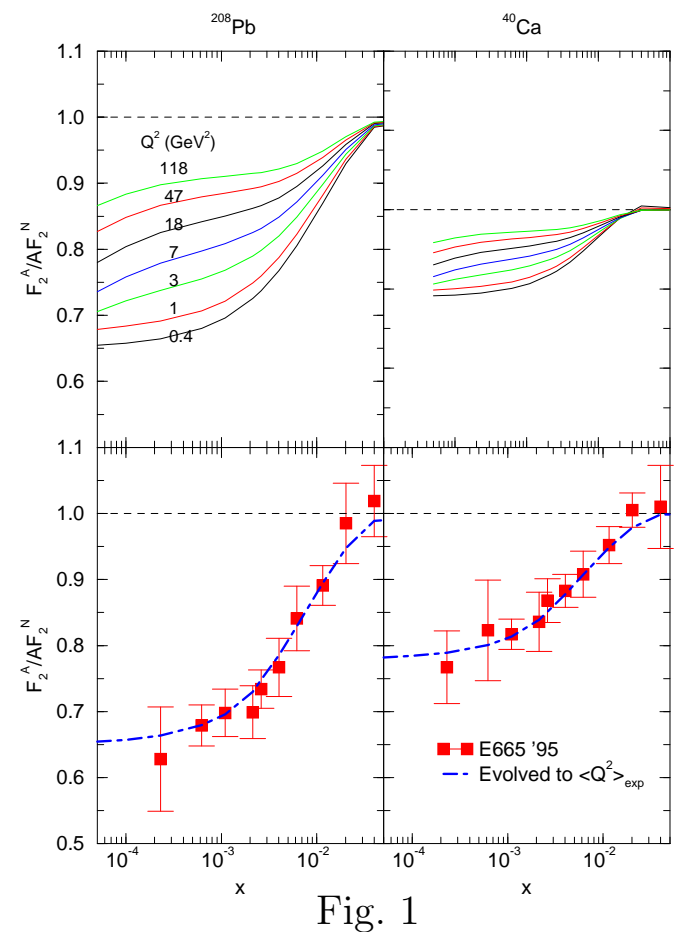

The essential difference between the quark and gluon cases is different splitting functions and the effective number of scatterings, $\kappa_{g}=9 \kappa_{q} / 4$ due to different color representations that the quark and the gluon belong to. These two effects combined result in the 12 times faster increase of the gluon density with $Q^{2}$ than in the case of the sea quarks in the region of small $x$. The two important effects which make the gluon shadowing quite different from the quark shadowing are the stronger scaling violation in the semihard scale region and a larger perturbative shadowing effect. This can be seen by considering the shadowing ratio

$$
R_{g}\left(x, Q^{2}\right)=\frac{x g_{A}\left(x, Q^{2}\right)}{A x g_{N}\left(x, Q^{2}\right)}=\frac{x g_{N}\left(x, Q_{0}^{2}\right) R_{g}^{0}(x)+\Delta x g_{A}\left(x ; Q^{2}, Q_{0}^{2}\right)}{x g_{N}\left(x, Q_{0}^{2}\right)+\Delta x g_{N}\left(x ; Q^{2}, Q_{0}^{2}\right)}
$$

where $R_{g}^{0}(x)$ is the initial shadowing ratio at $Q_{0}^{2}$ and $\Delta x g\left(x ; Q^{2}, Q_{0}^{2}\right)$ is the change of the gluon distribution as the scale changes from $Q_{0}^{2}$ to $Q^{2}$. The strong scaling violation due to a larger $\kappa_{g}$ at small $x$ causes $\Delta x g_{N}\left(x ; Q^{2}, Q_{0}^{2}\right) \gg x g_{N}\left(x, Q_{0}^{2}\right)$ as $Q^{2}$ is greater than $1 \sim 2$ $\mathrm{GeV}^{2}$. As seen in Fig. 2(a) for $Q^{2} \geq 3 \mathrm{GeV}^{2}$ the dependence of $R_{g}\left(x, Q^{2}\right)$ on the initial condition $R_{g}^{0}(x)$ diminishes and the perturbative shadowing mechanism takes over.

The $x$-dependence of the gluon shadowing can also be predicted as long as $Q^{2}>3 \mathrm{GeV}^{2}$ where the influence of the initial condition is minimal. The shape of the distribution is quite robust in the small- $x$ region regardless of what initial conditions one may choose. Due to the perturbative nature of the shadowing, these distributions do not exhibit a saturation as $x$ decreases. Furthermore since the shadowing is a non-linear effect in the effective number of scatterings, the impact parameter dependent shadowing ratio cannot be factorized into a product of an average shadowing ratio and the nuclear thickness function. Our results are presented in Fig. 2(b).

In summary, the nuclear shadowing phenomenon is a consequence of the parton coherent multiple scatterings. While the quark density shadowing arises from an interplay between "soft" physics and the semihard QCD process, the gluon shadowing is largely 


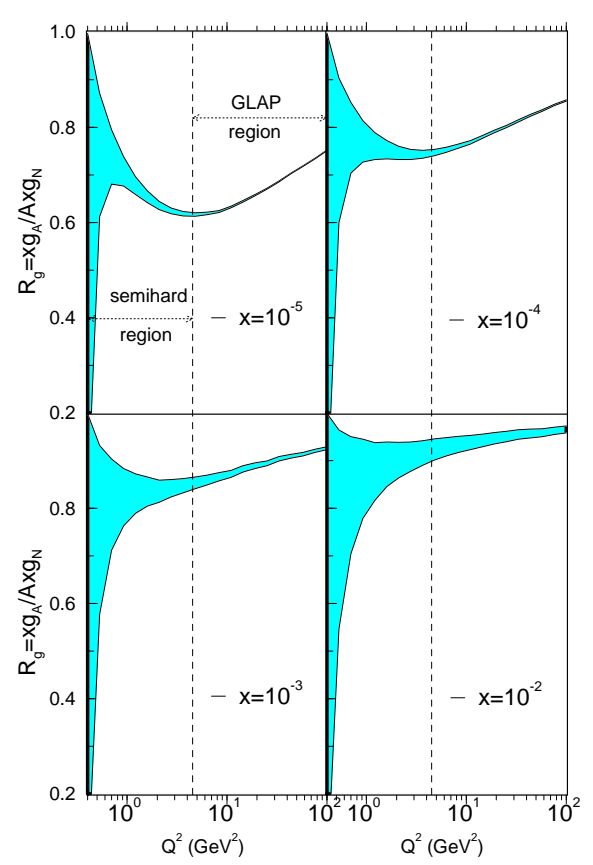

Fig. 2(a)
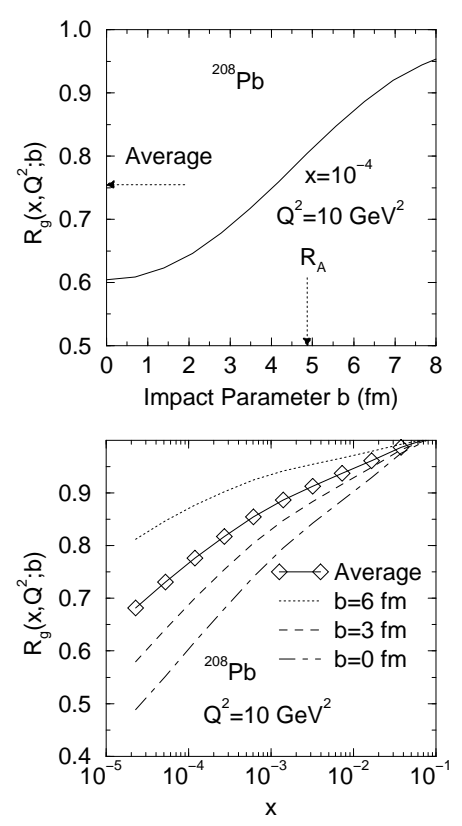

Fig. 2(b)

driven by a perturbative shadowing mechanism due to the strong scaling violation in the small- $x$ region. The gluon shadowing is thus a robust phenomenon at large $Q^{2}$ and can be unambiguously predicted by perturbative QCD. The strong scaling violation of the nucleon structure function in the semihard momentum transfer region at small $x$ can be reliably described by perturbative QCD and is a central key to the understanding of the scale dependence of the nuclear shadowing effect. The impact parameter dependence of gluon shadowing is a non-linear effect in the nuclear thickness function. It is important to correctly incorporate the impact parameter dependence of the nuclear structure function when one calculates the QCD processes in the central nuclear collisions.

\section{REFERENCES}

1. NMC Collaboration, P. Amaudruz et al., Z. Phys. C51, 387 (1991); Z. Phys. C53, 73 (1992); Phys. Lett. B295, 195 (1992); NMC Collaboration, M. Arneodo et al., Nucl. Phys. B441, 12 (1995).

2. E665 Collaboration, M.R. Adams et al., Phys. Rev. Lett. 68, 3266 (1992); Phys. Lett. B287, 375 (1992); Z. Phys. C67, 403 (1995).

3. D. Alde et al., Phys. Rev. Lett. 66, 133 (1991).

4. S. Gavin and J. Milana, Phys. Rev. Lett. 68, 1834 (1992).

5. see e.g., G. Piller, W. Ratzka and W. Weise, Z. Phys. A352, 427 (1995); T.H. Bauer, R.D. Spital and D.R. Yennie and F.M. Pipkin, Rev. Mod. Phys. 50, 261 (1978).

6. S.J. Brodsky and H.J. Lu, Phys. Rev. Lett. 64, 1342 (1990).

7. R.J. Glauber, in Lectures in theoretical physics, ed. W.E. Brittin et al. (Interscience Publishers, New York, 1959); R.J. Glauber and G. Matthiae, Nucl. Phys. B21, 135 (1970); V.N. Gribov, Sov. Phys. JETP 29, 483 (1969).

8. N.N. Nikolaev and B.G. Zakharov, Z. Phys. C64, 631 (1994).

9. Z. Huang, H. J. Lu and I. Sarcevic, hep-ph/9705250, submitted to Nucl. Phys. A. 10. A.L. Ayala F, M.B. Gay Ducati and E.M. Levin, Nucl. Phys. B493, 305 (1997). 